\title{
Análisis factorial exploratorio de una escala de gravedad de obstrucción bronquial evaluada en menores de tres años, Chile, 2015
} Exploratory factor analysis of a score for grading the severity
of bronchial obstruction evaluated in children younger than
3 years old, Chile, 2015

Klga. Mg. Soledad Luarte-Martínez ${ }^{a, b, f}$ y Enf. Mg. Paula Astudillo Díaz. ${ }^{b, c, d, e}$

a. Departamento de Kinesiología, Universidad de Concepción, Concepción, Chile.

b. Programa de Magíster en Ciencias Médicas, Universidad de La Frontera, Chile.

c. Departamento de Cirugía Traumatología y Anestesiología de la Universidad de La Frontera, Chile.

d. Programa de Doctorado en Ciencias Médicas, Universidad de La Frontera, Chile.

e. Programa de Doctorado en Psicología, Salud y Calidad de Vida, Universidad de Girona, España.

f. Grupo de Investigación en Salud Cardiovascular y Respiratoria (IDEAS-CVR), Concepción, Chile.

Correspondencia:

Enf. Mg. Paula Astudillo Díaz: paula.astudillo@ ufrontera.cl

Financiamiento: Financiado por la Vicerrectoría de Investigación y Desarrollo (VRID) de la Universidad de Concepción, proyecto N. ${ }^{\circ}$ 215.082.050IN.

Conflicto de intereses: Ninguno que declarar.

Recibido: 13-12-2016 Aceptado: 3-7-2017

\section{INTRODUCCIÓN}

En Latinoamérica, las infecciones Introducción. La escala de Tal modificada es utilizada en Chile para determinar la gravedad de la obstrucción bronquial. Existen informes de su validez; sin embargo, carece de antecedentes del análisis de su estructura como escala para cuantificar de forma significativa la gravedad de la obstrucción bronquial.

Objetivo. Identificar el patrón de relación entre los ítems de la escala y la dimensión gravedad de la obstrucción bronquial en una muestra de menores de tres años que consultan en dos centros de salud de la provincia de Concepción, Chile. Método. Análisis factorial exploratorio. Selección no aleatoria y voluntaria de participantes, menores de 36 meses, agosto-noviembre de 2015. Evaluados de manera independiente por médico y kinesiólogo, en tiempo seguido. Se aplican medidas de tendencia central, análisis factorial exploratorio, por subgrupo de menores y mayores de 6 meses, y consistencia interna. Resultados. 204 mediciones a 102 menores. Con una varianza explicada de $39 \%$, los ítems se dirigen a dos conceptos diferentes, un factor que agrupa frecuencia respiratoria, sibilancia y retracción, y otro que solo lo determina cianosis, con alfa de Cronbach de 0,5. Al extraer cianosis del análisis, muestra matriz monofactorial con $38 \%$ de varianza total explicada y consistencia interna de 0,62 .

Conclusiones. Por medio de sus ítems, la estructura dela escala noseajusta adecuadamente para esta muestra. Cianosis se muestra como un ítem disociado en la estructura. Al ajustar el modelo a solo tres ítems, la estabilidad del alfa como medida de consistencia interna aumenta, pero a un valor cuestionable, lo que, sin duda, variará al ser aplicada en otra muestra.

Palabras clave: análisis factorial exploratorio, enfermedades pulmonares obstructivas, escalas.

http:/ / dx.doi.org/10.5546/ aap.2017.e370

Texto completo en inglés:

http:/ / dx.doi.org/10.5546/ aap.2017.eng.e370

Cómo citar: Luarte-Martínez S, Astudillo Díaz P. Análisis factorial exploratorio de una escala de gravedad de obstrucción bronquial evaluada en menores de tres años, Chile, 2015. Arch Argent Pediatr 2017;115(6):e370-e376. respiratorias agudas (IRA) han sido la primera causa de consulta en atención primaria. ${ }^{1}$ Constituyen el mayor porcentaje de consultas anuales; en promedio, 56\% de IRA del tracto superior (IRA altas) y $44 \%$ de IRA del tracto inferior (IRA bajas), por lo que se convierten en la primera causa de hospitalización en pediatría. ${ }^{1-3}$

En Chile, en 1994, la neumonía era la primera causa de mortalidad infantil tardía..$^{2,3}$ El Ministerio de Salud (MINSAL) inició una estrategia con un programa unificado de IRA en el que se priorizaba la atención sobre la base de parámetros clínicos, lo que disminuyó las cifras de mortalidad infantil, desde 16 por cada 1000 nacidos vivos en 1990 a 7,9 por cada 1000 nacidos vivos en 2009.,3

En este contexto, para determinar la gravedad de la obstrucción bronquial (GOB), se han desarrollado diversas escalas de puntuación clínica. ${ }^{5,6}$ Dentro de estas, se encuentra la escala de Tal modificada, creada en 1974 por Bierman y Pearson. ${ }^{7}$ En 1983, fue modificada por $\mathrm{Tal}^{8}$ reemplazando la relación inspiración-espiración por cianosis; luego se incluyeron, en Chile, ${ }^{4}$ diferenciaciones por edad, y el MINSAL fue quien la recomendó a través de la "Guía de infecciones respiratorias".

Las validaciones de la escala de Tal incluyeron la de Pavón ${ }^{9}$ y mostraron buena correlación con la saturación de oxígeno $\left(\mathrm{SaO}_{2}\right)(\mathrm{r}=-0,76$; $95 \%$ intervalo de confianza [IC], de -0,83 a -0,68).

En 2007, Coarasa ${ }^{10}$ evaluó la capacidad para predecir la $\mathrm{SaO}_{2}$ 
menor de 91 o igual y mostró sensibilidad inferior a su homóloga argentina, y especificidad superior $(56 \%$ contra $100 \%$ y $92,6 \%$ contra $54,3 \%$, respectivamente; $\mathrm{p}<0,001)$. Puebla ${ }^{11}$ evaluó la utilidad diagnóstica por profesional sanitario no médico y obtuvo sensibilidad del 77\% (IC 95\%, $70 \%-82 \%$ ), especificidad de $88,4 \%$ (IC $95 \%$, $84 \%-92 \%$ ). McCallum ${ }^{12}$ evaluó la consistencia interna y confiabilidad de las escalas de Tal y Tal modificada, y aportó que ambas escalas contaban con adecuada consistencia interna (alfa de Cronbach de 0,66 y 0,7, respectivamente) y adecuada confiabilidad interobservador, kappa de 0,72 (IC 95\%: 0,63, 0,76) y 0,70 (IC 95\%: 0,63, $0,83)$, respectivamente.

Pese a la existencia de antecedentes del desempeño de la escala de Tal modificada como predictora de hipoxemia, es necesario que cuente con la evaluación de otros elementos de validez, ${ }^{13-15}$ como la evaluación de su estructura como escala, por medio de sus ítems, para confirmar que el fenómeno estudiado esté representado. Es un análisis poco habitual en salud, pero de relevancia como aporte a la validez, ya que el MINSAL de Chile la presenta como un modelo de medida ${ }^{3,16}$ que debe resumir la GOB de manera concreta.

Objetivo: Identificar el patrón de relación entre los ítems de la escala y la dimensión GOB en una muestra de menores de tres años, que consultaron en dos centros de salud de la provincia de Concepción, Chile.

\section{METODOLOGÍA}

Diseño: el análisis factorial exploratorio (AFE) es un modelo estadístico que representa las relaciones entre un conjunto de variables. Esta aproximación explorará patrones de relaciones entre la variable latente $(\mathrm{GOB})$ y variables manifiestas (ítems), identificará número y composición de variables necesarios para explicar la varianza común del conjunto de ítems analizado. La aproximación inductiva busca patrones de relación entre indicadores (ítems) y define el constructo (GOB). Esta manera de proceder en la perspectiva exploratoria puede tener implicancias importantes sobre las inferencias de los modelos teóricos, ${ }^{17}$ ya que trabaja desde las mediciones empíricas de los indicadores hacia la definición del constructo. Hace referencia a la capacidad de un instrumento de medición para cuantificar de forma significativa y adecuada el rasgo para cuya medición ha sido diseñado.

\section{Supuestos del diseño}

a. Cada variable observada o ítem está seleccionado para que refleje la característica del factor que se pretende medir, en este caso, GOB.

b. Personas con diferentes niveles en el factor común (GOB) darán diferentes respuestas a ese ítem, justamente porque el factor causa diferentes respuestas de ese ítem (el ítem es una manifestación de GOB).

Sujetos: el tamaño muestral se basó en las recomendaciones de Streiner ${ }^{18}$ que postulaba un número mínimo de 10 observaciones por ítem de escala para validar. Se consideró un mínimo de 40 observaciones.

Se realizó una selección no aleatoria y voluntaria (tutor) de los participantes, pacientes pediátricos de primera consulta en el Centro de Salud Víctor Manuel Fernández y Hospital Guillermo Grant Benavente de la provincia de Concepción entre agosto y noviembre de 2015. Criterios de inclusión: menores de 36 meses, con cuadro bronquial obstructivo (diagnosticado por el médico residente). Se excluyeron con patología pulmonar crónica de diagnóstico previo.

Recolección de datos: dos evaluadores independientes, médico y kinesiólogo (centro de salud), que realizaron evaluaciones en tiempo seguido (para evitar variaciones en los signos clínicos); asignaron puntaje a cada ítem y la suma total de la escala. Se realizó una preprueba para determinar tiempos e inconvenientes de evaluación en tiempo seguido. La gestión de base de datos se trabajó en el programa Access ${ }^{\circledR}$.

\section{Variables de la escala}

- Variables biodemográficas: sexo, edad (meses), escolaridad materna (último nivel aprobado), número del grupo familiar, asistencia a sala cuna-jardín infantil (sí/no). Datos obtenidos mediante una entrevista con el evaluador.

- Escala monofactorial de 4 ítems, frecuencia respiratoria, sibilancias, cianosis, retracción. Con puntaje tipo Likert; el valor " 0 " fue inexistencia de manifestación o manifestación en valores normales, y "3", manifestación de mayor riesgo:

- Frecuencia respiratoria, subdividida en menores de 6 meses ( $\leq$ a 40 respiraciones por minuto -rpm-, 41-55 rpm, 56-70 rpm, $>70 \mathrm{rpm})$ y en mayores de 6 meses o iguales ( $\leq 30 \mathrm{rpm}, 31-45 \mathrm{rpm}, 46-60 \mathrm{rpm},>60 \mathrm{rpm}$ ).

- Sibilancias (audibles a distancia, inspiración y espiración con fonendoscopio, fin de 
espiración con fonendoscopio, no existían).

- Cianosis (generalizada, perioral en reposo, perioral al llorar, no existía).

- Retracción (supraclavicular, intercostal, subcostal, no existía).

\section{Plan de análisis}

Se buscaron patrones de relación en términos de varianza. No se analizó la capacidad predictiva, sino que si ese ítem efectivamente tenía relación en términos de caracterizar la GOB.

Se buscaron medidas de tendencia central y distribución de frecuencias. Para el AFE, se extrajeron datos fuera de rango. Esto es un control de calidad antes de iniciar el análisis, se les llama "outliers". Los valores atípicos podían tener un efecto desproporcionado en los

TABLA 1. Variables sociodemográficas $(n=102)$

\begin{tabular}{lc}
\hline Variable & $\%$ \\
\hline Escolaridad materna & \\
$\quad$ Básica & 14,7 \\
$\quad$ Media & 59,8 \\
$\quad$ Superior & 25,5 \\
N. ${ }^{\circ}$ de personas por vivienda & \\
$\quad \geq 5$ & 32,4 \\
$\quad<5$ & 67,7 \\
Asistencia a sala cuna & \\
$\quad$ Sí & 33,3 \\
$\quad$ No & 66,7 \\
\hline
\end{tabular}

resultados estadísticos, lo que podía conducir a interpretaciones engañosas.

Para evaluar la pertinencia del AFE, se hizo una evaluación del supuesto de correlación entre variables. Para establecer si se justificaba o no su aplicación, se buscaron correlaciones entre cada ítem de la escala, aplicando correlación de Spearman (rho); se aplicó la medida de adecuación muestral de Kaiser-Meyer-Olkin $(\mathrm{KMO})$ y la prueba de esfericidad de Bartlett, y se planteó una conveniente adecuación muestral en valores sobre 0,6 .

Posteriormente, concibiendo el error, se utilizó un método de extracción de ejes principales y rotación de varimax para llegar a la matriz factorial; luego se analizó la consistencia interna (CI) con alfa de Cronbach; se trabajó bajo recomendaciones de George y Mallery: ${ }^{19}$ mayor de 0,7 , aceptable; mayor de 0,6 , cuestionable, y mayor de 0,5, pobre. Se trabajó en el programa SPSS $11^{\circledR}$.

Se analizaron matrices para $\leq 6$ meses y $>6$ meses.

\section{Aspectos éticos involucrados en la investigación}

Los tutores de pacientes participaron de manera voluntaria, con la firma previa del consentimiento. Aprobado (Código CEC:15-0740) por el Comité de Ética del Servicio de Salud de Concepción, Chile, en agosto de 2015.

TABla 2. Descripción según frecuencias de las variables de la escala y diferencias de proporciones según el evaluador

\begin{tabular}{|c|c|c|c|c|}
\hline Variable & Categoría & Médico $(n=102)$ & Kinesiólogo $(n=102)$ & Valor de $p$ \\
\hline $\mathrm{FR} \leq 6$ meses & $\begin{array}{l}\leq 40 \mathrm{rpm} \\
41-55 \mathrm{rpm} \\
56-70 \mathrm{rpm} \\
>70 \mathrm{rpm}\end{array}$ & $\begin{array}{c}1 \\
18 \\
87 \\
1\end{array}$ & $\begin{array}{c}8 \\
12 \\
13 \\
1\end{array}$ & 0,5925 \\
\hline $\mathrm{FR}>6$ meses & $\begin{array}{c}\leq 30 \mathrm{rpm} \\
31-45 \mathrm{rpm} \\
45-60 \mathrm{rpm} \\
>60 \mathrm{rpm}\end{array}$ & $\begin{array}{c}23 \\
38 \\
13 \\
4\end{array}$ & $\begin{array}{c}24 \\
36 \\
15 \\
3\end{array}$ & 0,4 \\
\hline Sibilancias & $\begin{array}{c}\text { No } \\
\text { Fin de espiración c/ fonendo } \\
\text { Inspiración y espiración c/ fonendo } \\
\text { Audibles a distancia }\end{array}$ & $\begin{array}{c}34 \\
51 \\
12 \\
5\end{array}$ & $\begin{array}{c}30 \\
53 \\
13 \\
6\end{array}$ & 0,4698 \\
\hline Cianosis & $\begin{array}{c}\text { No } \\
\text { Perioral al llorar } \\
\text { Perioral en reposo } \\
\text { Generalizada en reposo }\end{array}$ & $\begin{array}{c}100 \\
2 \\
0 \\
0\end{array}$ & $\begin{array}{c}102 \\
0 \\
0 \\
0\end{array}$ & 0,0001 \\
\hline Retracciones & $\begin{array}{c}\text { No } \\
\text { Subcostal }(+) \\
\text { Intercostal }(++) \\
\text { Supraclavicular }(+++)\end{array}$ & $\begin{array}{c}41 \\
41 \\
14 \\
5\end{array}$ & $\begin{array}{c}45 \\
41 \\
12 \\
4\end{array}$ & 0,0049 \\
\hline
\end{tabular}

FR: frecuencia respiratoria. 


\section{RESULTADOS}

\section{Análisis descriptivo}

Se completaron 204 observaciones (mediciones) de escala a 102 pacientes pediátricos, 42 niñas $(41,2 \%)$ y 60 niños $(58,8 \%)$. Mediana de edad de 10 meses (1-35 meses); 67,7\% vivía con menos de 5 personas en el hogar; $66,7 \%$ no asistía regularmente a sala cuna, por lo que se encontraba al cuidado de padres o cuidadores; $85,3 \%$ de las madres contaba con escolaridad entre media y superior (Tabla 1).

En la distribución de frecuencias de variables de la escala de Tal modificada y diferencias de proporciones según el evaluador, el valor " $p$ " en cianosis mostró la probabilidad de que existieran diferencias; sin embargo, estas podían estar relacionadas con la ausencia de un mayor número de casos graves recolectados (Tabla 2).

En relación con el puntaje total de la escala en mayores de 6 meses y menores de 6 meses, la GOB fue leve (Tabla 3).

\section{Análisis factorial exploratorio}

Se buscaron valores fuera de rango calculando rango, desviación estándar, promedio individual, coeficiente de variación Mahalanobis, y quedaron 179 mediciones. Con estas 179, se realizó el AFE.

El AFE se inició con el cálculo de correlaciones de rho de Spearman (Tabla 4).

En la matriz factorial, con una varianza explicada de $39 \%$, se observaron dos factores, uno agrupó frecuencia respiratoria, sibilancia y

TABLA 3. Resultados de la escala de Tal modificada

\begin{tabular}{lcc}
\hline Variable & $\mathbf{6}$ meses $(\mathrm{n}=\mathbf{2 6})$ & $\mathbf{> 6}$ meses $(\mathbf{n}=\mathbf{7 6})$ \\
\hline Frecuencia. respiratoria & $1(0-2)$ & $1(0-3)$ \\
Sibilancias & $1(0-2)$ & $1(0-3)$ \\
Cianosis & $0(0-0)$ & $0(0-1)$ \\
Retracciones & $1(0-2)$ & $1(0-3)$ \\
Puntaje total & $3(0-6)$ & $3(0-9)$ \\
\hline
\end{tabular}

Los resultados se expresan en mediana y rango.

TABLA 4. Correlaciones de Spearman entre cada uno de los items de la escala ( $\mathrm{n}=179$ mediciones)

\begin{tabular}{lcccc}
\hline & $\begin{array}{c}\text { Frecuencia } \\
\text { respiratoria }\end{array}$ & Sibilancias & Cianosis & Retracciones \\
\hline Frecuencia & & & & \\
respiratoria & 1 & 0,23 & $-0,17$ & 0,47 \\
Sibilancias & 0,23 & 1 & $-0,79$ & 0,24 \\
Cianosis & $-0,17$ & $-0,79$ & 1 & 0,10 \\
Retracciones & 0,47 & 0,24 & 0,01 & 1 \\
\hline
\end{tabular}

retracción, y otro factor, cianosis. Ya teniendo correlaciones bajas y, además, fijando un solo factor, el ítem cianosis se disoció en relación con otros ítems, sin explicar el constructo GOB. Se calculó CI de 0,5 (Tabla 5).

En vista de los resultados, se realizó una nueva matriz que excluía cianosis del análisis, y se obtuvo una matriz monofactorial (que explicaba el constructo GOB) y los ítems frecuencia respiratoria, sibilancias y retracción con $38 \%$ de la varianza total. Las cargas factoriales obtenidas de frecuencia respiratoria, sibilancias y retracciones fueron 0,64 , 0,4 y 0,75 , respectivamente. Se determinó CI con estos 3 ítems, con un valor de 0,62.

\subsection{Análisis factorial exploratorio en menores de 6 meses}

Con 46 mediciones, se realizó el cálculo de correlaciones de Spearman, en el que cianosis no se correlacionaba con ningún ítem, presentaba varianza cero, por tanto, no se podían extraer datos para el AFE. Se analizó excluyendo cianosis del análisis y se mostró una matriz con varianza total de $41 \%$, monofactorial, que agrupaba frecuencia respiratoria, sibilancias y retracción, con cargas factoriales de 0,5, 0,6 y 0,67, respectivamente. El CI de estos 3 ítems tenía valor 0,7 .

\subsection{Análisis factorial exploratorio en mayores de 6 meses o iguales}

Con 133 mediciones, se analizó la matriz y se observó que cianosis poseía carga factorial hacia

TABla 5. Matriz factorial de los cuatro items de la escala ( $n=179$ mediciones $)$

\begin{tabular}{lcc}
\hline & \multicolumn{2}{c}{ Factor } \\
& $\mathbf{1}$ & $\mathbf{2}$ \\
\hline Frecuencia respiratoria & 0,62 & \\
Sibilancias & 0,38 & \\
Cianosis & & 0,50 \\
Retracciones & 0,82 & \\
\hline
\end{tabular}

Alfa de Cronbach $=0,5$

Tabla 6. Mayores de 6 meses o iguales: matriz factorial de los 4 items de la escala ( $n=133$ mediciones)

\begin{tabular}{lcc}
\hline & \multicolumn{3}{c}{ Factor } \\
& $\mathbf{1}$ & $\mathbf{2}$ \\
\hline Frecuencia respiratoria & 0,62 & \\
Sibilancias & 0,34 & 0,54 \\
Cianosis & & \\
Retracciones & 0,84 & \\
\hline
\end{tabular}

Alfa de Cronbach $=0,52$ 
otro factor (Tabla 6). Se extrajo cianosis del análisis, y se obtuvo una nueva matriz monofactorial que agrupaba frecuencia respiratoria, sibilancias y retracción con cargas factoriales de $0,65,0,37$ y 0,75, respectivamente, que presentaba una $\mathrm{CI}$ de 0,6 .

\section{DISCUSIÓN}

Entre las características sociodemográficas de la muestra, la mayoría poseía madres con escolaridad media y universitaria, y no vivían en hacinamiento, lo que había cambiado en relación con los inicios del programa IRA en Chile, cuyas características, en función de baja educación materna o hacinamiento, incrementaban el riesgo de morir por neumonía, una complicación de cuadros bronquiales obstructivos. ${ }^{11}$

Respecto a la escala, la mayor diferencia de proporciones según el evaluador fue en cianosis y retracciones. Probablemente, se debió a la subjetividad de ambos signos: eran observables no cuantificables, mientras que, para frecuencia respiratoria, se realizaba un conteo en tiempo determinado y, para sibilancias, se utilizaba un instrumento (fonendoscopio).$^{20}$ La evaluación de cianosis y retracción dependía de la observación, método subjetivo indirecto. ${ }^{21}$

Respecto a la frecuencia y rango de respuesta, los niños y niñas a quienes se aplicó la prueba calificaron igual un ítem, por tanto, existía la posibilidad de que el ítem cianosis no estuviera aportando variabilidad a la escala ${ }^{14}$ por tratarse de un signo de presentación tardía respecto a los otros ítems.

En el AFE, se observó que, entre los ítems de la escala, existía uno que se disociaba, lo que podría explicarse porque cianosis era un signo tardío de dificultad respiratoria, que aparecía cuando la hemoglobina desoxigenada en sangre alcanzaba entre 4 y 6 g/ dl; las $\mathrm{SaO}_{2}$ de, aproximadamente, 80-87\% darían lugar a cianosis clínicamente aparente..$^{22}$ Este signo clínico podría no aportar información a la estructura de la escala, ya que, al extraerlo, se determinaba un solo constructo con un aumento en la consistencia; sin embargo, esta magnitud de consistencia interna aportaba valores de cuestionables a aceptables. Es necesario considerar que esta estimación es bajo el supuesto de datos continuos y podría estar subestimada. ${ }^{23,24}$

Las matrices obtenidas para análisis por subgrupo presentaron el mismo comportamiento respecto a cianosis, y se ajustaron los ítems frecuencia respiratoria, sibilancias y retracciones. Esto se explicaría por lo referido anteriormente en función del signo clínico cianosis como signo tardío, ya que eran prematuras la gran mayoría de las consultas por cuadros bronquiales obstructivos.

Comparando la matriz factorial por subgrupo de edad sin el ítem cianosis, se observó que todas las cargas factoriales en los menores de 6 meses entregaban valores sobre 0,6 al constructo GOB. Esto podría explicarse ya que, entre menos edad tuviera el niño, mayor resistencia e inestabilidad presentaría su vía aérea, ${ }^{25}$ hiperreactiva ante cualquier cuadro infeccioso en coherencia con los signos característicos que componían la escala.

Se podría pensar que los meses de recolección de datos, entre agosto y noviembre de 2015, podrían haber afectado la tasa típica de consulta de época invernal; sin embargo, en los últimos 5 años, las IRA han presentado un comportamiento bimodal, y se han destacado picos de consultas entre junio y agosto de 2015 (el mayor número ha sido entre los meses de agosto y septiembre del presente año). ${ }^{5}$

Respecto a los antecedentes de la validez de criterio, en el estudio de McCallum y cols., ${ }^{12}$ observaron bajo rendimiento diagnóstico de esta escala. Coarasa y cols., ${ }^{10}$ mostraron una sensibilidad notoriamente inferior a su homóloga de Argentina. Además, Pavón y cols., ${ }^{9}$ observaron que el ítem cianosis de la escala de Tal modificada mostraba menor nivel de correlación con la saturación periférica. Si bien son evaluaciones que no tienen que ver con el objetivo del estudio, parecen estar en coherencia respecto al comportamiento de la escala como estructura.

Los criterios utilizados en este diseño por sí mismos suponen solo una aproximación a la validación del constructo GOB para esta muestra de niños y niñas; por ello, es necesario complementarlo con un análisis factorial confirmatorio (AFC), que es una estrategia sumamente útil en el ámbito de la prueba de hipótesis y la confirmación de teorías. ${ }^{26,27} \mathrm{Sin}$ embargo, no se pudo realizar, principalmente, por la ausencia de multicolinealidad (no se presenta una fuerte correlación entre variables explicativas del modelo).

En cuanto a las limitaciones del trabajo: 1) el tamaño de la muestra puede ser adecuado de acuerdo con recomendaciones teóricas, pero estuvo compuesto preferentemente por niños y niñas del perímetro urbano. Para futuros trabajos, debe procurarse utilizar muestras representativas de la población general, como pacientes rurales o de diferentes sectores geográficos, y discriminar entre distintas poblaciones. 2) La gran mayoría 
de la muestra estudiada presentó un puntaje equivalente a la categoría de obstrucción bronquial leve, y esto no permitió conocer la correlación entre las variables en su nivel más alto de gravedad.

Como aspectos positivos del estudio, el AFE ha sido riguroso y esta aproximación exploratoria, aunque no es, en absoluto, suficiente para llegar a hacer una modificación de la escala actualmente vigente, reúne información valiosa y relevante para considerar la generación de hipótesis y nuevos estudios, así como también reflexiones teóricas y empíricas sobre la medición de la GOB.

Como recomendaciones: a) realizar estudios que repliquen los resultados obtenidos con niños y niñas en diferentes contextos socioculturales, indagando variabilidad del ítem cianocis; b) realizar análisis factorial confirmatorio, junto con medidas de invarianza para evaluar la sensibilidad al cambio o discriminar entre distintas poblaciones; c) realizar estudios para conocer cómo progresa la GOB. La teoría que subyace al constructo GOB puede aportar información en esta línea, estudios longitudinales que analicen empíricamente la relación antecedentes y resultados de los ítems de la escala.

\section{CONCLUSIONES}

Para esta muestra, el fenómeno estudiado no está representado adecuadamente por sus ítems. Por medio de sus ítems, la estructura de la escala no se ajusta de manera apropiada.

Para el subgrupo de menores de 6 meses, la estructura de la escala no se ajusta adecuadamente. El modelo de estructura se muestra con valores de consistencia interna aceptable y solo involucraría frecuencia respiratoria, sibilancia y retracción. El ítem cianosis se muestra como un ítem disociado en la estructura. Al ajustar el modelo a solo tres ítems, la estabilidad del alfa como medida de consistencia interna aumenta, pero muestra un valor cuestionable, lo que, sin duda, variará al ser aplicada en otra muestra.

\section{REFERENCIAS}

1. OMS. Medidas de control de infecciones en la atención sanitaria de pacientes con enfermedades respiratorias agudas en entornos comunitarios. Guía para el instructor. OMS; 2009. [Acceso: 5 de julio de 2017]. Disponible en: http: / / apps.who.int/iris / bitstream/10665/70233/1/ WHO_HSE_GAR_BDP_2009.1_spa.pdf.

2. Chile. Ministerio de Salud. Consultas de morbilidad por enfermedades respiratorias en salas IRA, ERA y Mixta, por Región y Servicio de salud, SNSS 2014. Departamento de Estadística e Información de Salud. 2014. [Acceso: 5 de julio de 2017]. Disponible en: http://intradeis. minsal.cl / reportesrem / 2014 / REM23SECCIOND / REM23SECCIOND.aspx.

3. Chile. Ministerio de Salud. Guía Clínica Auge Infección Respiratoria Aguda Baja de Manejo ambulatorioen menores de 5 años. 2013. [Acceso: 4 de julio de 2017]. Disponible en: http: / / www.minsal.cl/ portal/url/item/ $7220 f d c 4341244$ a9e04001011f0113b9.pdf.

4. Girardi GB, Astudillo PO, Zúñiga FH. El programa IRA en Chile: hitos e historia. Rev Chil Pediatr 2001;72(4):292-300.

5. Bekhof J, Reimink R, Brand PL. Systematic review: Insufficient validation of clinical scores for the assessment of acute dysponea in wheezing children. Pediatr Respir Rev 2014;15(1):98-112.

6. Luarte-Martínez S, Rodríguez-Núñez I, Astudillo P, et al. Propiedades psicométricas de las escalas de gravedad de obstrucción bronquial en pediatría. Revisión sistemática y metaanálisis. Arch Argent Pediatr 2017;115(3):241-8.

7. Bierman CW, Pierson WE. The Pharmacologic Management of Status Asthmaticus in Children. Pedriatrics 1974:54;245-7.

8. Tal A, Bavilski C, Yohai D, et al. Dexamethasone and Salbutamol in the Treatment of Acute Wheezing in Infants. Pediatrics 1983;71(1):13-8.

9. Pavón D, Castro-Rodríguez JA, Rubilar L, et al. Relation between pulse oximetry and clinical score in children with acute wheezing less than 24 months of age. Pediatr Pulmonol 1999;27(6):423-7.

10. Coarasa A, Giugno H, Cutri A, et al. Validación de una herramienta de predicción clínica simple para la evaluación dela gravedad en niños con síndrome bronquial obstructivo. Arch Argent Pediatr 2010;108(2):116-23.

11. Puebla Molina S, Bustos L, Valenzuela M, et al. La escala de Tal como test diagnóstico y el diagnóstico clínico como gold standard en el síndrome bronquial obstructivo del lactante. Rev Pediatr Aten Primaria 2008;10(37):45-53.

12. McCallum GB, Morris PS, Wilson CC, et al.Severity scoring systems: are they internally valid, reliable and predictive of oxygen use in children with acute bronchiolitis? Pediatr Pulmonol 2013;48(8):797-803.

13. Alarcón A, Muñoz S. Medición en Salud: Algunas consideraciones metodológicas. Rev Med Chile 2008;136(1):125-30.

14. Sánchez R, Echeverry J. Validación de escalas de medición en salud. Rev Salud Pública 2004;6(3):302-18.

15. Abell N, Springer DW, Kamata A. Developing and validating rapid assessment instruments. Oxford: Oxford University Press; 2009.

16. McDowell I, Newell C. The Theoretical and technical Foundations of Health Measurement. En:Measuring Health: a guide to rating scales and questionnaires. $2^{\text {nd }}$ ed. New York: Oxford University Press; 1996.Págs.10-46.

17. Gerbin DW, Hamilton JG. Viability of Exploratory Factor. Analysis as a Precursor to Confirmatory Factor Analysis. Struct Equ Modeling 1996;3(1):2-72.

18. Streiner DL, Norman GR. Health measurement scales: A practical guide to their development and use. 4th ed. Oxford, UK: Oxford University Press; 2008.

19. George D, Mallery M. SPSS for Windows Step by Step: A Simple Guide and Reference 21.0.13th ed. Boston: Allyn \& Bacon/Prentice Hall; 2013.

20. Sánchez I. Aplicaciones clínicas del estudio objetivo de los ruidos respiratorio en pediatría. Rev Chil Pediatr 2003;74(3):259-68.

21. Cruz M. Aparato respiratorio. En: Nuevo Tratadode Pediatría. 2. ${ }^{\text {da }}$ ed. Barcelona: Océano-Ergón; 2011.Págs.1420-32.

22. Steinhorn RH. Evaluation and management of the cyanotic neonate. Clin Pediatr Emerg Med 2008;9(3):169-75. 
e376 / Arch Argent Pediatr 2017;115(6):e370-e376 / Artículo original

23. George D, Mallery P. SPSS for Windows step by step: A simple guide and reference. 11.0 update. 4th ed. Boston: Allyn \& Bacon; 2003.

24. Oliden PE, Zumbo BD. Coeficientes de fiabilidad para escalas de respuesta categórica ordenada. Psicothema 2008;20(4):896-901.

25. Rodríguez I, Báez C, Contreras T, et al. Kinesioterapia respiratoria en la bronquiolitis aguda: estrategia terapéutica, bases fisiológicas e impacto clínico. Neumol Pediatr
2013;8(3):111-5.

26. Batista Foguet JM, Coenders G, Alonso J. Análisis factorial confirmatorio. Su utilidad en la validación de cuestionarios relacionados con la salud. Med Clín (Barc) 2004;122(Supl 1):21-7.

27. Batista Foguet JM, Coenders Gallart G. Modelos de ecuaciones estructurales: modelos para el análisis de relaciones causales. Cuadernos de estadística. 2. da ed. Madrid: La Muralla; 2000. 